\title{
Prediction of Vortex-Induced Vibration of Bare Cylinder and Cylinder Fitted with Helical Strakes
}

\author{
KeeQuen Lee ${ }^{1}$, Aminudin Abu ${ }^{1}$, Pauziah Muhamad ${ }^{1}$, Asnizah Sahekhaini ${ }^{2}$, Litken $\operatorname{Tan}^{1}$ and HooiSiang Kang ${ }^{3}$ \\ ${ }^{1}$ Malaysia - Japan International Institute of Technology (MJIT), University Teknologi Malaysia Kuala Lumpur, Malaysia \\ ${ }^{2}$ Politeknik Sultan Salahudin Abdul Aziz Shah, 40150 Shah Alam, Malaysia \\ ${ }^{3}$ Institute for Vehicle Systems \& Engineering (IVeSE), Faculty of Mechanical Engineering, Universiti Teknologi Malaysia, Johor Bahru, \\ Malaysia
}

\begin{abstract}
In the study, the vortex-induced vibration (VIV) of a cylinder fitted with and without helical strakes is investigated using fluid-structure interaction (FSI) software. The purpose is to predict the VIV characteristic of cylinder fitted with and without helical strakes. Fluid and structural solvers in commercial computational fluid dynamic (CFD) software were implemented as two-way FSI solvers to develop the simulation. A flexible circular cylinder of diameter, $\mathrm{D}=0.018 \mathrm{~m}$ was tested. The pitch and height of the helical strakes were $10 \mathrm{D}$ and $0.10 \mathrm{D}$, respectively. Existing experiment outputs of bare cylinder were used as benchmark to verify the simulated results. In overall, the simulation was able to predict the trend of the amplitude response and the mean drag coefficient. However, a slight over-prediction was noticed. It was also found that the helical strakes was able to reduce up to $60 \%$ of the VIV in water.
\end{abstract}

\section{Introduction}

Marine riser, which is one of the important components in the offshore structure, play a crucial role in extracting the hydrocarbon products from undersea drilling site to the oil platform. Due to the unpredictable sea current, risers face several problems such as creeping, buckling, expansion and fatigue generated by vortex-induced vibration (VIV). Among these problems, VIV, which can be explained as the vibration stimulated by the external fluid flow vortices when interacting with the structure, is one of the critical design issue of the riser. Currently most of the investigations of the VIV of riser are conducted experimentally [1-4]. Nevertheless, due to the high cost in constructing the experimental facilities and large aspect ratio of the riser, several factors are usually being constrained in the model testing. This initiates the exploration of fluid-structure interaction (FSI) software in predicting the VIV characteristic of a riser. Although FSI software is widely used in several applications such as combustion system $[5,6]$ and arterial blood flow $[7,8]$, its involvement on flow pass a circular riser/cylinder in three-dimensional is still rare. Several studies using twodimensional (2D) FSI simulation are found. Pereira Gomes et al. [9] conducted a 2D FSI simulation on an aluminium cylinder in a laminar flow. The outputs were acceptable, but deficiencies were observed due to the constraint of damping coefficient. Facchinetti et al. [10] utilized classical van der Pol equation with equation of motion to examine VIV of a rigid cylinder in 2D. The authors recommended that the van der Pol wake oscillator was suitable in 2D simulation and was in high potential to be used in 3D analysis. Gustafsson [11] was one of the few who conducted 3D FSI simulation to analyse the VIV of a short riser. The author pointed that time step was an important factor to decide the simulation's accuracy. Besides, he explained that FSI simulation was much more time consuming compare to the pure computational fluid dynamic (CFD) simulation. Therefore, high level of mesh refining was unrealistic as it increases the computational time.

On the other hand, the simulation of the application of helical strakes on a circular cylinder has not been widely explored. Constantinides and Oakley [12] were the very few researchers that investigate the flow around a cylinder fitted with helical strakes. The authors observed that the VIV suppression mechanism was lack of spanwise correlation, and found that the vortices formation was farther downstream.

To the author knowledge, there is still no published work using FSI simulation to predict the response of cylinder fitted with helical strakes. Since the reviews have shown that FSI study on riser is very limited and preliminary, we hope to explore its capability in predicting the VIV characteristic of cylinder fitted with and without helical strakes in three-dimension. The existing experimental results of bare cylinder - cylinder without fitted with helical strakes [13] will be used as benchmark to validate the simulation outputs.

\section{Computational study}




\subsection{Fluid equation}

Fluid-structure interaction, a combination of fluid and structural solvers, is implemented in the study. The unsteady Reynolds-averaged Navier-Stokes equations (RANS) is used as the governing equation for the fluid. For an incompressible Newtonian fluid, the equation is expressed as below:

$$
\begin{gathered}
\frac{\partial U_{i}}{\partial x_{i}}=0 \\
\rho \frac{\partial U_{i}}{\partial t}+\rho U_{j} \frac{\partial U_{i}}{\partial x_{j}}=-\frac{\partial p}{\partial x_{i}}+\frac{\partial}{\partial x_{j}}\left(2 \mu S_{j i}-\rho \overline{u_{j}^{\prime} u_{i}^{\prime}}\right)
\end{gathered}
$$

where $U$ is the averaged velocity, $u$ ' as the fluctuating velocity, $\mathrm{P}$ denotes pressure, $\rho$ represents density, and $\mathrm{Sji}$ and $\mu$ are rate of strain tensor and molecular viscosity, respectively. Reynolds-stress tensor is represented by the term . At this stage, the system is not closed because of the number of equations are less than the number of unknown components. Hence, additional equations are needed. In the study, k- $\omega$ based Shear Stress Transport (SST) eddy viscosity turbulence model is chosen as this model considers the turbulent shear stress, which is not accounted in other turbulence model. Therefore, it is able to estimate the separation flow under adverse pressure gradient more precisely [19].

\subsection{Structural equation}

To couple with fluid response, equation of motion is utilized as structural dynamic system for a cylinder in the study. A spring-damper-mass system with external fluid forces for a cylinder is described as:

$$
m_{s} \ddot{\chi}+c_{s} \dot{\chi}+k_{s} \chi=F_{\text {fluid }}(t)
$$

where ms denotes the structure mass, cs as the damping coefficient, ks as the spring stiffness and Ffluid(t) represents the forces created by fluid on the cylinder body. The fluid force is obtained from the pressure and body viscous friction based on Navier-Stokes equation solutions. $\chi=\mathrm{Xi}+\mathrm{Yj}+\mathrm{Zk}$, where $\mathrm{X}, \mathrm{Y}$ and $\mathrm{Z}$ is the instantaneous displacement of the cylinder in $\mathrm{x}, \mathrm{y}$ and $\mathrm{z}$ direction, respectively. The first and second order-partial derivative of the displacement are denoted as $\dot{\chi}$ and $\ddot{\chi}$, respectively.

\subsection{Coupling for fluid and structure}

The concept of FSI is to coupling the equation of fluid and structure, as shown in Figure 1. The system coupling is a two-way coupling. Partitioned approach is implemented as suitable solver because it allows the selection of each domain based on the requirement. The concept of the coupling is as following: Firstly, to achieve converged solution, the fluid calculation is executed. The calculated fluid forces of the fluid interface are then inserted to the structural domain. In the structural domain, the hydrodynamic forces are used as the boundary conditions to further compute the deformation of structural mesh. The deformation output is the cylinder displacement. Then, the displacements information is sent back to the fluid domain to recalculate the change of hydrodynamic forces. The process is repeated until the predetermined limits are reached for both displacement and force values.

\section{Boundary condition}

\subsection{Structural domain}

The cylinder is modelled to be similar to a pre-tensioned flexible cylinder, where both ends of the cylinder are pinned, and one the cylinder's end is allowed to move in axial direction. To achieve that, at the ends of the cylinder, all of the displacements and rotation in $\mathrm{Z}$ direction are set to be zero. However, rotation is allowed in $\mathrm{x}$ and $\mathrm{y}$ directions. For the cylinder end where axial movement is allowed, instead of applying zero in $\mathrm{z}$ direction, an axial force of $147 \mathrm{~N}$ is used to represent the pre-tensioned effect. The gravity effect of the cylinder is represented by applying a standard earth gravity in $-Y$ direction. Function of Fluid Solid Interface is defined at the cylinder surface to allow the integration of force and displacement between structural and fluid domains.

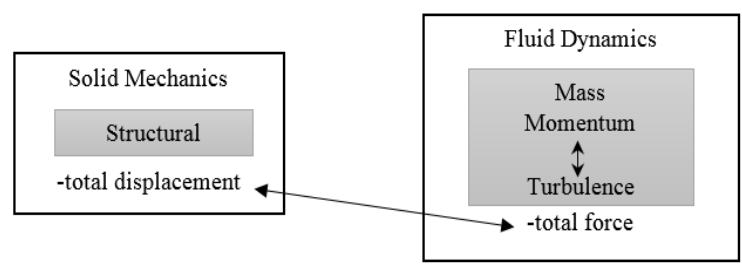

Figure 1. Coupling between structural and fluid equations.

\subsection{Fluid domain}

The fluid domain is consists of seven boundaries, namely upstream and downstream boundaries, left and right boundaries, upper and lower boundaries and the cylinder wall. In deciding the size of the computational domain, the distance between cylinder and boundaries is very important to avoid the effect of side boundaries [14]. According to Borch and Rodi [15], the minimum distance should be $4.5 \mathrm{D}$ to evade the influence of pressure and velocity, where $\mathrm{D}$ denotes the diameter of the cylinder. The authors recommended that boundary distance of 10D was appropriate to eliminate the effect. Hence, distance of 10D from the cylinder is set for upstream, lower and upper boundaries, as shown in Figure 2. To ensure the vortices of the cylinder can be captured successfully without any disturbance from the downstream boundary, distance of $20 \mathrm{D}$ is used between the cylinder and downstream boundary.

\subsection{Grid independence study}

Three different level of meshing sizes, namely coarse, medium and fine meshing are tested to check the 
sensitivity of the mesh towards the output of the simulation. The mesh size should be appropriate to avoid mesh dependency outputs. The grid independence study is tested on a fix end short cylinder. The outputs are presented in terms of cross-flow (CF) amplitude response, as shown in Table 1.

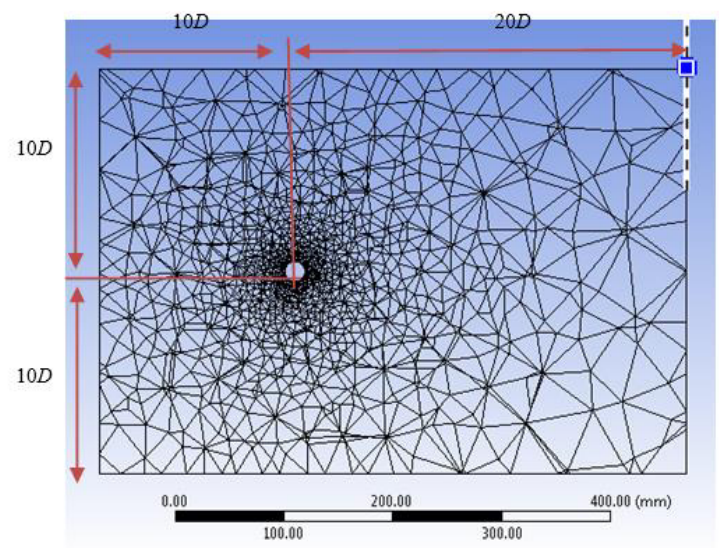

Figure 2. Cylinder mesh in fluid domain.

Table 1. Amplitude response of different mesh size.

\begin{tabular}{|c|c|c|c|}
\hline $\begin{array}{c}\text { Meshing } \\
\text { size }\end{array}$ & $\begin{array}{c}\text { Number of } \\
\text { elements } \\
\left(\times 10^{5}\right)\end{array}$ & $\begin{array}{c}\text { Amplitude } \\
\text { response } \\
(\mathrm{m})\end{array}$ & $\begin{array}{c}\text { Discrepancy } \\
\text { of amplitude } \\
\text { (standard } \\
\text { deviation) }\end{array}$ \\
\hline Coarse & 11.6 & 0.0162 & $13 \%$ \\
\hline Medium & 11.7 & 0.0143 & $2.5 \%$ \\
\hline Fine & 11.8 & 0.0140 & - \\
\hline
\end{tabular}

Based on table 1, the discrepancy of amplitude in term of standard deviation is relatively minor in overall. The difference between the coarse and fine mesh is around $13 \%$, while only $2.5 \%$ of changes in amplitude is found between the medium and fine mesh. This indicates that the mesh sensitivity especially for medium and fine meshing is adequate and independent from the change of mesh size. To minimize the total computational time, medium mesh size is selected for the rest of the simulations. Table 2 shows the dimensions and the summary of computational meshes used for bare cylinder and cylinder fitted with helical strakes. Only strakes with pitch (p) of 10D and height (h) of 0.10D is tested in the study.

\section{Result and discussion}

The purpose of conducting the simulation is to estimate the ability of the FSI software in predicting the response of a flexible cylinder. Only lock-in region is investigated in the study as it is the most critical region that required more attention. Lock-in is defined as the occurrence of large amplitude oscillation due to the synchronization of structural vibration frequencies and the vortex shedding [16]. The simulation is run for cylinder fitted with and without helical strakes. The existing experimental results of bare cylinder with similar configuration [13] are used as for comparison.
Table 2. Summary of computational meshes used.

\begin{tabular}{|l|c|c|c|}
\hline \multicolumn{1}{|c|}{$\begin{array}{c}\text { Type of } \\
\text { cylinder }\end{array}$} & $\begin{array}{c}\text { Length, } \\
L(\mathrm{~m})\end{array}$ & $\begin{array}{c}\text { Diameter } \\
, D(\mathrm{~m})\end{array}$ & $\begin{array}{c}\text { Number of } \\
\text { elements } \\
\left(\mathrm{x} 10^{5}\right)\end{array}$ \\
\hline Bare & 2.92 & 0.018 & 11.7 \\
\hline $\begin{array}{l}\text { Helical strakes } \\
(p=10 D ; h= \\
0.10 D)\end{array}$ & 2.92 & 0.018 & 13.2 \\
\hline
\end{tabular}

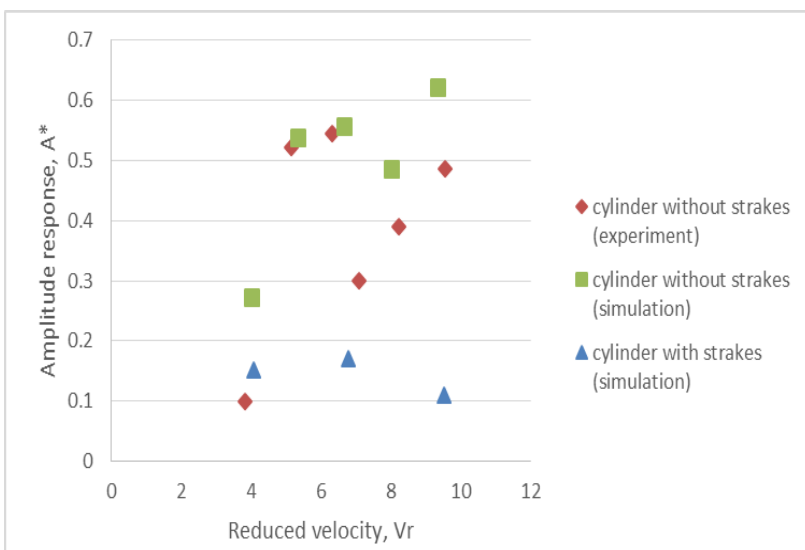

Figure 3. Amplitude response of cylinder fitted with and without helical strakes.

Figure 3 indicates the amplitude response of the simulated and experimental results. Five cases of different reduced velocity are tested for bare cylinder in simulation. The reduced velocity is defined as

$$
V_{r}=\frac{U}{f_{n} D}
$$

where $\mathrm{D}$ is the diameter of cylinder, fn is the natural frequency and $U$ as the flow velocity. In overall, the simulated bare cylinder results follow the trend of the experimental results. Nevertheless, the values are slightly overvalued. Another three runs of simulations on cylinder fitted with helical strakes show apparent amplitude reduction from 0.5 in average to less than 0.2 . This shows that the application of helical strakes is able to reduce the amplitude and hence the vibration of the cylinder. The VIV suppression efficiency ratio of the helical strakes of the present study is 82.5 percent, which can calculated by:

efficiency ratio $=\frac{\left(\frac{A}{D}\right)_{\max _{\_} \text {bare }}-\left(\frac{A}{D}\right) \max _{\text {matrakes }}}{\left(\frac{A}{D}\right) \max _{\text {bare }}} \times 100 \%$

where $(\mathrm{A} / \mathrm{D}) \max$ indicates the maximum amplitude ratio. Percentage as high as $93.1 \%$ is reported by Boubenider et al. [17]. The output indicates that FSI is able to capture the response of a cylinder fitted with and without helical strakes.

Figure 4 shows the mean drag coefficient of the simulated and experimental results. Similar to the amplitude response, the mean drag coefficient of the simulated bare cylinder is slightly overestimated compare to the experimental output. However, the graph trend is the same, where the mean drag coefficient increases 
gradually until $\mathrm{Vr} \approx 5.5$ and then slowly decreases to lower values. The mean drag coefficient of the simulated cylinder fitted with helical strakes, on the other hand, is found to be higher than the bare cylinder. The same condition is reported by Gomez et al. [18] where the installation of helical strakes increases the drag force of a cylinder. The mean drag coefficient of helical strakes remains around value of 3 without influence of the reduced velocity. It is reasonable because the vortex behind the cylinder has been disrupted by the helical strakes, hence the change of the reduced velocity does not create different patterns of vortices that can affect the drag coefficient. The constant values of drag coefficient is also as reported by Boubenider et al. [17] in their study.

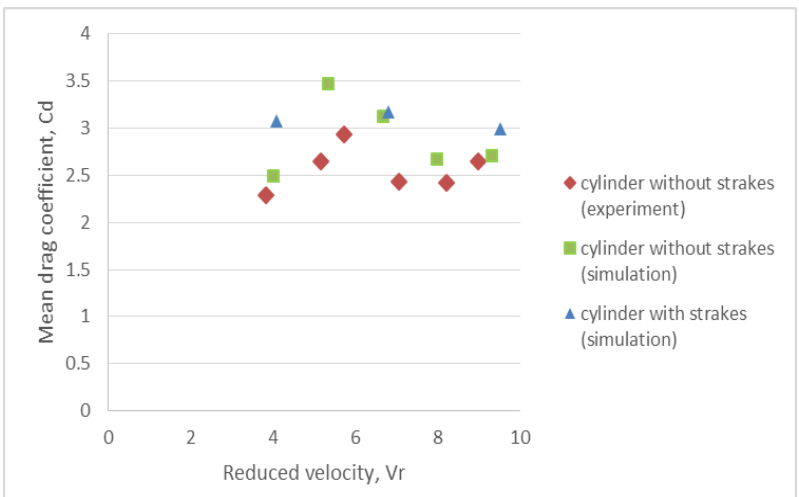

Figure 4. Mean drag coefficient of cylinder fitted with and without helical strakes.

\section{Conclusion}

The purpose of the study, which is to test the capability of FSI simulation in predicting the VIV characteristic of cylinder fitted with and without helical strakes, is done successfully. Fluid and structural solvers are used as twoway FSI solvers to develop the simulation. In general, minor over-prediction is found when comparing the simulated outputs with the experimental data. However, the graph trends are similar. Significant amplitude reduction and slightly increase of mean drag coefficient are observed after the application of helical strakes on the cylinder. The findings are in agreement with previous research works. Further work should be conducted to improve the over-prediction of the outputs.

\section{Acknowledgements}

This research was financially supported by Universiti Teknologi Malaysia under Potential Academic Staff research grant (Q.K130000.2743.02K26).

\section{References}

1. Y. Gao, S. Fu, T. Ren, Y. Xiong, L. Song, Appl. Ocean Res. 52, 102 (2015)

2. S. Huang, A. Sworn 2013. Appl. Ocean Res. 43, 21 (2013)

3. F.J. Huera-Huarte, Z.A. Bangash, L.M. Gonzalez J. Fluid Struct 48, 81 (2014)

4. J.R. Chaplin, P.W. Bearman, F.J. Huara Huarte, R.J. Pattenden. J. Fluids Struct. 21, 3 (2005)

5. Z. Khatir, A.K. Pozarlik, R.K. Cooper, J. W. Watterson. Int. J. Numer. Method Fluid, 1 (2006)

6. M. Shahi, J.B.W. Kok, P.R. Alemela. Proceedings ASME Turbo Expo. Copenhagen, Denmark (2012)

7. C.A. Figueroa, I.E. Vignon-Clementel, K.E. Jansen, T.J.R. Hughes, C.A Taylor. Comput. Method Appl. M. 195, 5685 (2006)

8. J.F. Gerbeau, M. Vidrascu, P. Frey. Comput. Struct. 83, 155 (2005)

9. J. Pereira Gomes, S. Yigit, H. Lienhart, M. Schafer. J. Fluid Struct 27, 43 (2011)

10. M.L. Facchinetti, E. Langre, F.. J. Fluid Struct. 19, 123 (2004)

11. A. Gustafsson. Master thesis. Chalmers University of Technology, Sweden (2012)

12. Y. Constantinides and O.W. Oakley. Proceedings of OMAE, 25 ${ }^{\text {th }}$ International Conference on Offshore Mechanics and Arctic Engineering, Germany (2006)

13. L.K. Quen, A. Abu, N. Kato. Appl. Ocean Res. 44, 82 (2014)

14. T. Farrant, M. Tan, W.G. Price. Comput. Fluid 30, $211(2001)$

15. G. Bosch and W. Rodi. Int. J. for Numer. Method Fluid 28, 601 (1998)

16. R. Bourguet, G.E. Karniadakis, M.S. Triantafyllou. J. Fluid Struct 27, 838 (2011)

17. R. Boubenider, K. Alptunaer, P. Fourchy, J.J. Wilde. Offshore Technology Conference in Houston, Texas (2008)

18. F. Gómez, J.H. Quesada, R. Gómex, V. Theofilis, B. Carmo, J. Meneghini. 43 ${ }^{\text {rd }}$ AIAA Fluid Dynamics Conference, San Diego, CA. (2013)

19. F.R. Menter,. AIAA J. 32, 8, 1598 (1994) 